\title{
Maternal vitamin D status-not the key to offspring bone health?
}

M aternal vitamin $\mathrm{D}$ status in utero is unlikely to be a key determinant of a child's bone health, report a team of UK researchers.

Pregnant women have been targeted as a specific group for whom vitamin D screening and supplementation could be important, partly because maternal vitamin $\mathrm{D}$ status in pregnancy has been suggested to influence the bone mineral content (BMC) of offspring. Studies of this association have, however, been limited by small sample sizes and produced inconsistent findings, including positive, negative and null associations. Lawlor and co-workers conducted a prospective study to find out whether maternal 25-hydroxyvitamin D levels affect the BMC of offspring 9-10 years after birth.

"Our aim was to conduct the largest study to date of the association between maternal vitamin D status and BMC in offspring," says lead researcher Debbie Lawlor from the University of Bristol. "We studied just under 4,000 motheroffspring pairs, a sample size that was over 10 times larger than the combined number of individuals assessed in all previous studies. We also tested whether the association was mainly seen in relation to mothers' 25 -hydroxyvitamin D levels measured in the third trimester of pregnancy (rather than earlier in pregnancy), as it had been suggested that it is during this time that a biological effect would be expected to occur."

The study included 3,960 mother-andsingleton-offspring pairs from the Avon Longitudinal Study of Parents and Children (ALSPAC); most of the mothers were of white European ethnicity. Measurements of levels of 25-hydroxyvitamin D during pregnancy were available for 1,035 (26\%) of women in their first trimester, $879(22 \%)$ of women in their second trimester and 2,046 (52\%) of women in their third trimester. Concentrations of 25-hydroxyvitamin D of $\geq 50 \mathrm{nmol} / \mathrm{l}$ were classified as sufficient,
49.99-27.50 $\mathrm{nmol} / \mathrm{l}$ as insufficient and $>27.50 \mathrm{nmol} / \mathrm{l}$ as deficient. Offspring $\mathrm{BMC}$ of the total body less the head and at the spine was measured by dual-energy $\mathrm{X}$-ray absorptiometry (DXA) scans at ages 9-10 years (mean 9.9 years); other bone outcomes were also measured, including BMD and bone area.

Of the pregnant women, around twothirds $(2,644 ; 67 \%)$ had sufficient vitamin D levels, 1,096 (28\%) had insufficient levels and $220(6 \%)$ were vitamin D deficient. The levels of 25-hydroxyvitamin D in the mothers and their offspring at ages 9-10 years were weakly associated.

The main finding of the study was that maternal 25-hydroxyvitamin D levels during any trimester of pregnancy were not associated with offspring BMC at ages 9-10 years. Offspring of mothers with insufficient and deficient levels of vitamin D had similar mean BMC as offspring of mothers with sufficient vitamin D levels. Furthermore, maternal 25-hydroxyvitamin D levels were not associated with the other bone outcomes studied. "We undertook a lot of sensitivity analyses and from these conclude that there really is no evidence that a mother's vitamin D status in pregnancy is important for her offspring's bone health," comments Lawlor. She suggests that "women should not be advised that their vitamin D level in pregnancy is important for their child's future bone health." The researchers note, however, that only one aspect of the effects of vitamin $\mathrm{D}$ in pregnant women was assessed in their study.

Cyrus Cooper from the Universities of Southampton and Oxford, UK, who was not involved in the study, points out that the findings "appear at first sight to be at variance with data previously published by the same group using the same cohort". Lawlor et al. concede that a previous ALSPAC study that reported an association between maternal ultraviolet $B$ (UVB) radiation exposure in the third

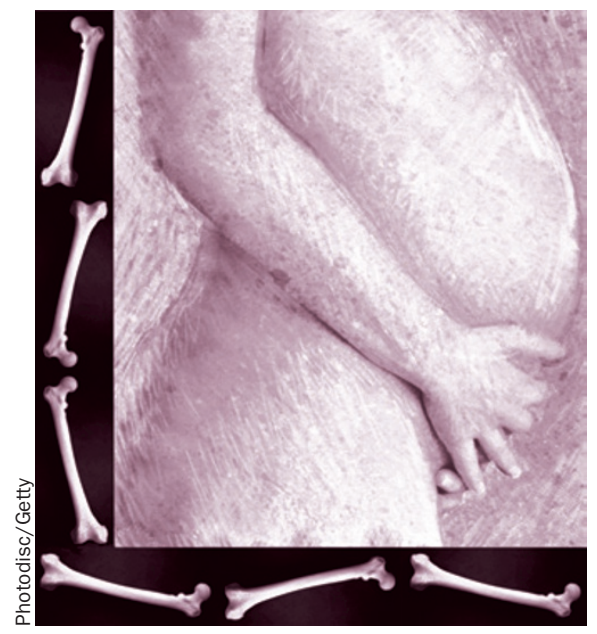

trimester of pregnancy and offspring BMC at a mean age of 9.9 years had an unidentified confounding factor: DXA measurements were carried out at an older age in offspring of mothers who had been in their third trimester during the summer months, when UVB levels are high. In regard to vitamin $\mathrm{D}$ supplementation during pregnancy, Cooper holds that "clearly further studies are required".

Uncertainty about the health benefits of vitamin $\mathrm{D}$ is much wider than that involving maternal vitamin D status and offspring bone health and needs to be resolved, Lawlor stresses. "All of the evidence concerning vitamin D and health outcomes needs to be reviewed so that the public and health professionals are clear about its health benefits and the levels of vitamin $\mathrm{D}$ that are considered normal in different people at different times in their lives."

\section{Carol Wilson}

This article originally appeared in Nat. Rev. Endocrinol. (doi:10.1038/nrendo.2013.63).

\footnotetext{
Original article Lawlor, D. A. et al. Association of maternal vitamin $\mathrm{D}$ status during pregnancy with bone-mineral content in offsrping: a prospective cohort study. Lancet doi:10.1016/S0140-6736(12)62203-X
} 